\title{
Gradhiva
}

GRADHI

Revue d'anthropologie et d'histoire des arts

13 | 2011

Pièges à voir, pièges à penser

\section{Jean-Hubert Martin et la pensée visuelle}

Entretien

Jean-Hubert Martin, Carlo Severi et Julien Bonhomme

\section{CpenEdition}

Journals

Édition électronique

URL : http://journals.openedition.org/gradhiva/2120

DOI : $10.4000 /$ gradhiva. 2120

ISSN : 1760-849X

Éditeur

Musée du quai Branly Jacques Chirac

Édition imprimée

Date de publication : 18 mai 2011

Pagination : 130-147

ISBN : 978-2-35744-042-5

ISSN : 0764-8928

Référence électronique

Jean-Hubert Martin, Carlo Severi et Julien Bonhomme, « Jean-Hubert Martin et la pensée visuelle », Gradhiva [En ligne], 13 | 2011, mis en ligne le 18 mai 2014, consulté le 02 mai 2019. URL : http:// journals.openedition.org/gradhiva/2120; DOI : 10.4000/gradhiva.2120

(c) musée du quai Branly 


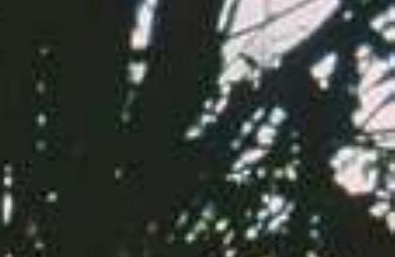

H.
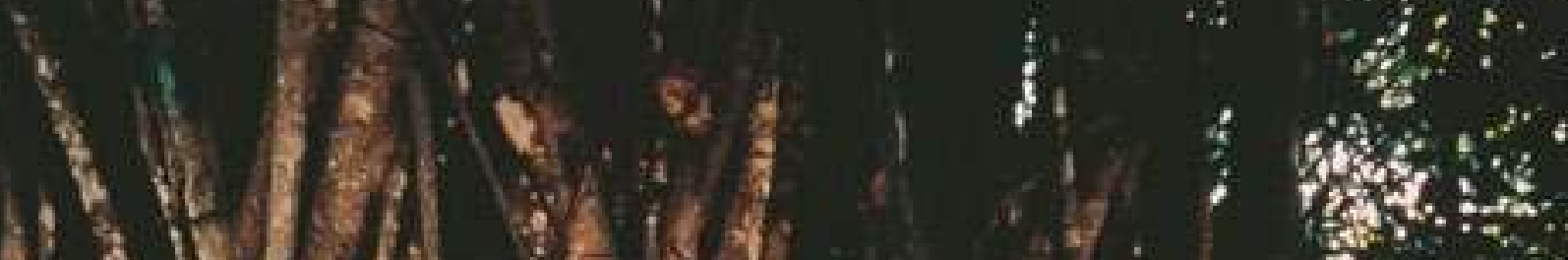

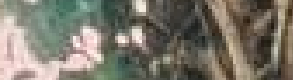
tian

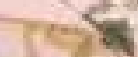

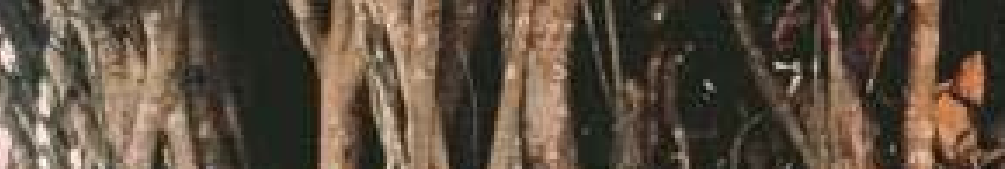

. Now - -1$)(2)$ $4.5(4 \times m) \times(m)$

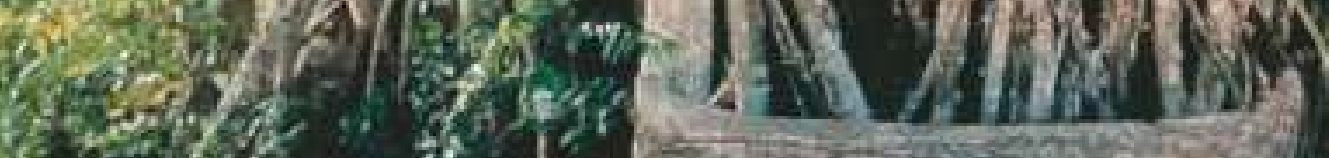

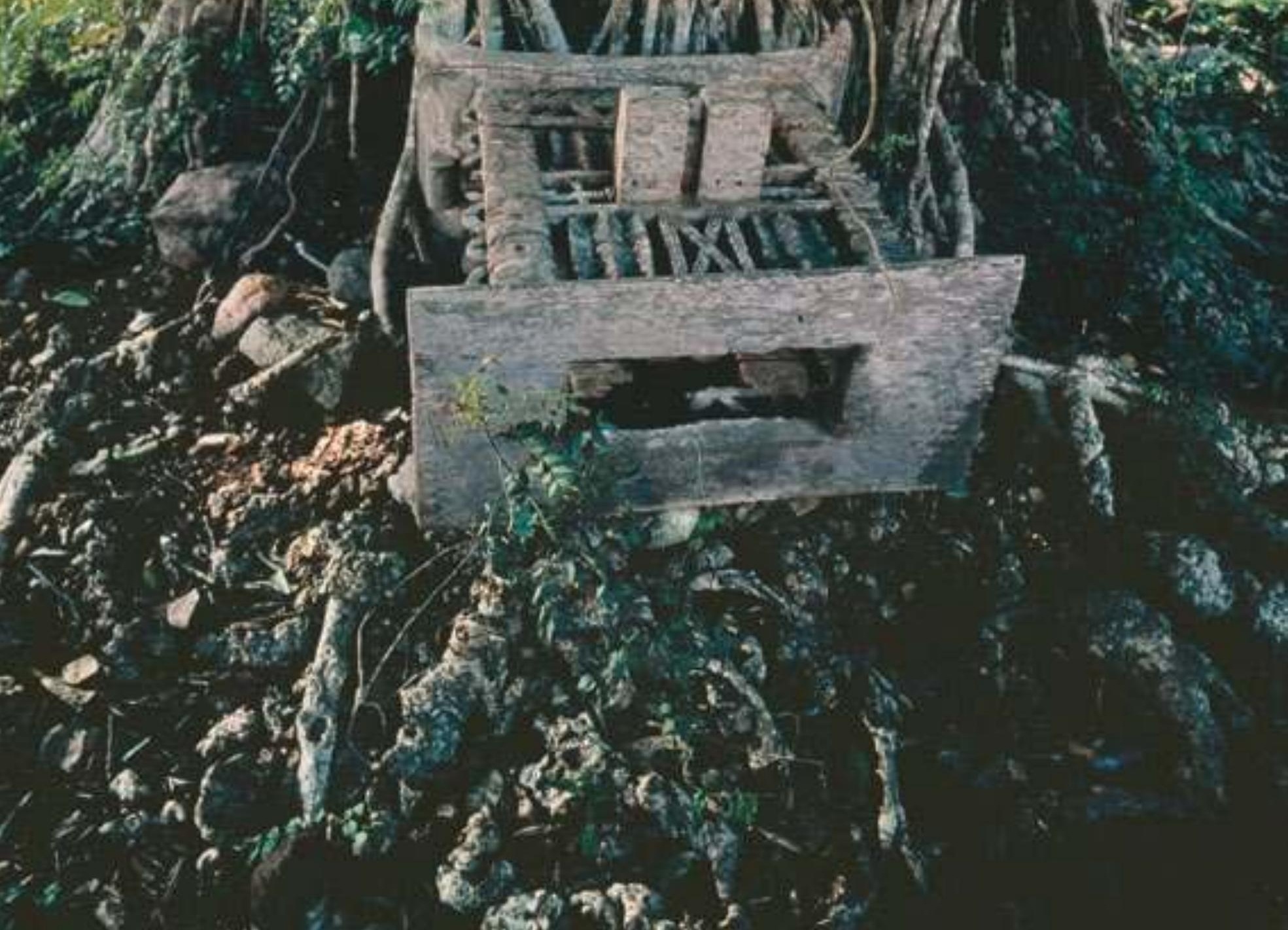




\section{Jean-Hubert Martin et la pensée visuelle}

Entretien Jean-Hubert Martin Carlo Severi Julien Bonhomme

-

1. Paris-Berlin,

12 juillet-6 novembre 1978 ,

Centre Georges-Pompidou, Paris.

2. Jean-Hubert Martin était commissaire invité de Partage d'exotisme, la $5^{\mathrm{e}}$ Biennale d'art contemporain de Lyon, du 27 juin au 24 septembre 2000.

3. Magiciens de la terre, 18 mai -28 août 1989, Centre GeorgesPompidou. Catalogue éponyme publié par les éditions du Centre Georges-Pompidou, Paris, 1989.
CARLO SEVERI : Votre travail a profondément marqué la présence des arts non occidentaux dans l'art contemporain. J'ai été frappé par cette déclaration, dans l'un de vos textes: "La nécessité d'ouvrir le cercle fermé de l'art contemporain occidental me hante depuis plusieurs années." C'est sur ce projet d'ouvrir le champ des possibles dans l'art contemporain que je voudrais axer notre conversation. Vous avez beaucoup fait discuter les anthropologues mais, paradoxalement, vous n'en avez pas bousculé les recherches; vous avez plutôt dérangé leurs esthétiques implicites, souvent rudimentaires. À propos de la mise en place d'une exposition, vous dites : «Une exposition, ça se bâtit en combinant mentalement des œuvres. " Vous explicitez ainsi le travail d'une pensée visuelle. Voilà donc quel pourrait être le thème de notre conversation: "Jean-Hubert Martin et la pensée visuelle».

En 1978, avec Werner Spies, vous avez dirigé l'exposition Paris-Berlin ${ }^{1}$ au Centre Pompidou. On pourrait se demander s'il existe une relation entre cette exposition et ce que vous avez entrepris par la suite. On est frappé par le fait que, dans votre itinéraire, vous n'avez pas emprunté les sentiers battus. D'autres historiens de l'art auraient pu, par exemple, s'inspirer de Nolde, Schmidt-Rottluff, Kirchner etc., et ensuite passer d'une vision primitiviste expressionniste à l'art primitif. Or, dans votre itinéraire intellectuel, on trouve tout autre chose. Dans le catalogue de la Biennale de Lyon², vous déclarez: "En fait, dans Dada, il y avait déjà, d'une part, l'idée de prôner l'égalité des œuvres et d'autre part, celle de se battre contre le culte héroïque de l'artiste. Le programme de Magiciens de la terre était là. » Magiciens de la terre aurait donc été inspiré par Dada? 


\section{PIÈGES À VOIR, PIÈGES À PENSER}

PAGE 130

Fig. 1 " Autel du tabouret » du Omanhene (roi) d'Anomabu et de ses objets personnels (à la mort d'un roi, son tabouret personnel est rapporté auprès de la racine de l'arbre, lieu d'où il est issu et tire sa force spirituelle) @ akg-images/ Werner Forman.

\footnotetext{
- $\bigcirc$

4. Une image peut en cacher une autre. Arcimboldo, Dalí, Raetz, 8 avril-6 juillet 2009, Galeries nationales du Grand Palais. Catalogue éponyme de l'exposition publié par la Rmn, Paris, 2009.

5. «Est-Ouest, Nord-Sud : nouveaux venus, nouveaux continents », in Daniel Soutif (éd.), L'Art du $x x^{e}$ siècle 1939-2002 : de l'art moderne à l'art contemporain . Paris, Citadelles et Mazenod, 2005 : 459-478.
}

Julien Bonнomme : Vous dites également, dans le catalogue d'Une image peut en cacher une autre 4 que vous êtes parti de l'image double du dadaïsme et du surréalisme pour aller en chercher ailleurs. Dada apparaîtrait donc bien comme un fil directeur.

JEAN-HUBERT MARTIN : La réponse est absolument oui. Cependant, je ne vois pas immédiatement le parallèle que vous tirez entre Paris-Berlin et les expositions que j'ai pu monter par la suite. Ce n'est pas nécessairement faux, mais il s'agit d'une reconstruction a posteriori. Quand on étudie un artiste, on essaie toujours d'établir une cohérence. Elle est souvent reconstruite par l'artiste lui-même ou par les autres, et tout le monde en est satisfait. Mais, c'est aussi très intéressant de voir son parcours comme un chaos, comme une suite de ruptures. Par rapport aux Magiciens de la terre et à la phrase que vous citez, j'ai, en vingt ans, nécessairement évolué dans mes propres interprétations et j'ai sans doute dit dans des interviews des choses différentes. Or, justement, que les Magiciens de la terre soient partis d'une idée dadaïste, je me suis privé de le dire au début parce que l'exposition, comme vous le savez, était vivement attaquée et j'ai tu cette filiation dadaïste. En revanche, je l'ai dit dans des interviews plus récentes. Je crois même l'avoir écrit dans un ouvrage collectif dirigé par Daniel Soutif sur le $\mathrm{xx}^{\mathrm{e}}$ siècle's. Il m'avait demandé un texte pour expliquer le contexte dans lequel j'ai élaboré Magiciens de la terre. Effectivement, on a cru que l'exposition était animée par cette "générosité " des Occidentaux riches de leurs moyens matériels et de leurs connaissances qui appellent de temps en temps un " autre » à participer à notre grand concert intellectuel et artistique. On retrouve en partie cela, mais cela ne constituait pas le moteur essentiel. Le moteur, c'était de mettre des artistes inconnus sur un pied d'égalité avec des célébrités. Si l'on cherche une espèce de continuité dans mon travail, cette idée me tient vraiment à cœur et je l'ai toujours défendue. Pour prendre une parabole, il y en a une très belle dans Magiciens de la terre, donnée par Braco Dimitrijevic, avec sa petite histoire du roi qui va à la chasse. Il y a deux artistes qui habitent l'un à côté de l'autre, le chien s'échappe et on retrouve le chien dans l'atelier de Léonard de Vinci et pas dans celui de l'autre artiste, dont on a même oublié le nom. Dans l'histoire de l'art, il y a beaucoup de hasards de ce genre. Pour ma part, j'aime bien m'intéresser à des artistes un peu oubliés, qui ne sont pas dans le mainstream, et conserver ce regard indépendant du marché, des médias, de la mode, etc., autant que l'on peut, pour analyser les choses sur le plan formel. L'histoire de l'art est intéressante quand elle tente de déterminer la part des inventions de formes.

Carlo SEVeri : Il y a une radicalité du mouvement Dada que vous développez, finalement, par une sorte de bataille continuelle, qui devient un défi de tous les jours. Le point de départ Dada, on le voit a posteriori, est important. Or, pour nous anthropologues, c'est une chose essentielle, c'est déjà toute une attitude par rapport au travail de l'artiste. Dans cette perspective, on suit les pas de l'artiste plutôt que de vouloir expliquer son œuvre; c'est probablement là quelque chose de très important pour l'anthropologie de l'art.

Je voulais aussi que l'on réfléchisse à ce qui vous sépare exactement de Rubin et de son exposition sur le primitivisme. Il y a probablement à la fois un enjeu intellectuel et un enjeu poétique dans cette opposition. Rubin est un moderniste classique. Il s'inspire du cubisme du Picasso des années 1920-1930, et ne possède pas la radicalité de quelqu'un qui s'inspire de Dada. On est donc tentés d'opposer cette dernière attitude, attitude de critique incessante des œuvres et de multiplication 


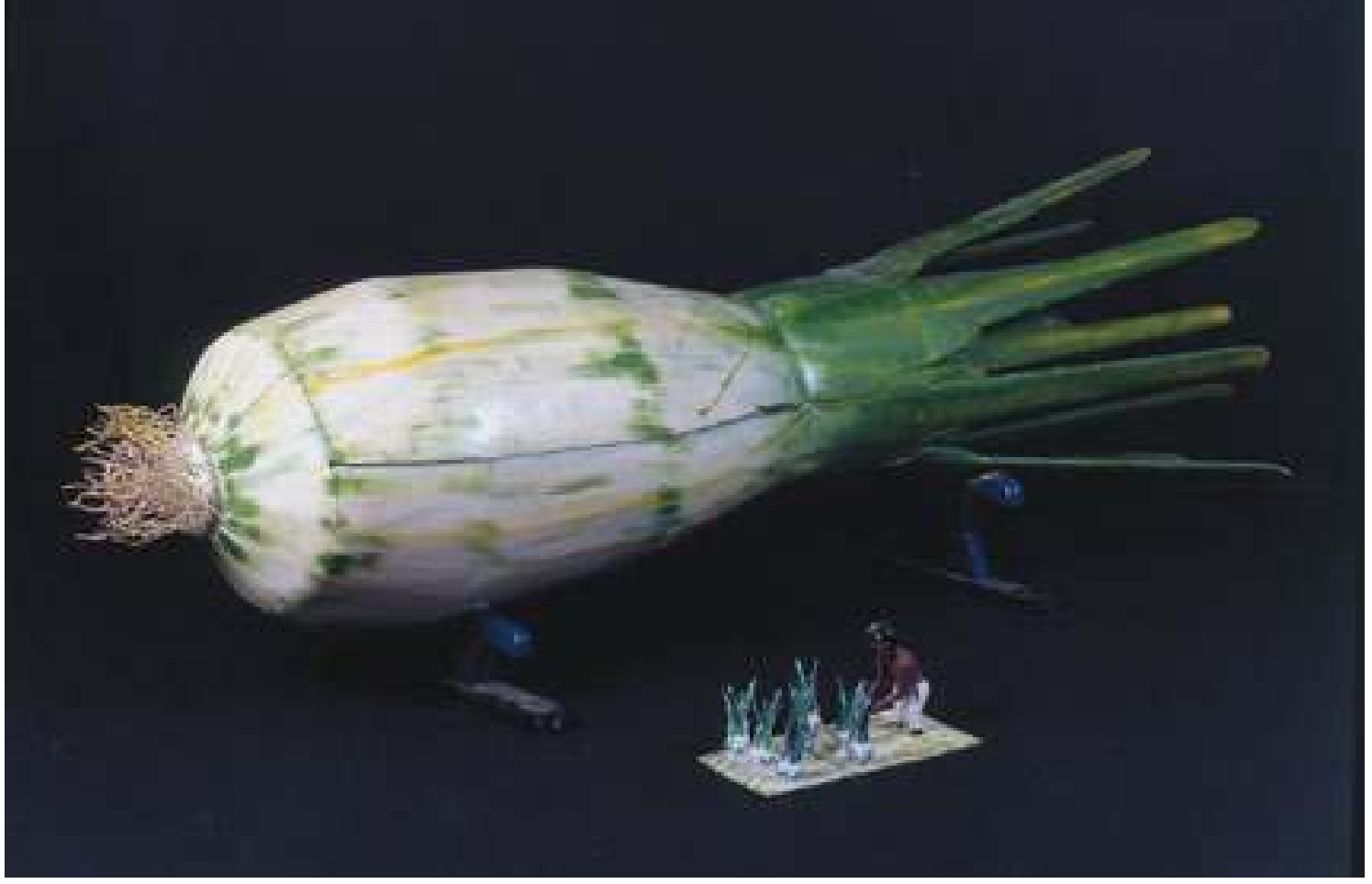

des frontières, à celle de Rubin qui a même essayé, dans une page impardonnable, d'appliquer les catégories de Riegl à l'Océanie et à l'Afrique. Dans le catalogue de Primitivism in $X^{\text {th }}$ century $^{6}$, Rubin parle en effet d'art tactile en Océanie et d'art optique en Afrique. J'aurais aimé avoir votre avis sur cette attitude.

JEAN-HUbert MARTIN : Évidemment, on m'a beaucoup opposé à Rubin. J'ai souvent dit que Magiciens de la terre était complémentaire de son exposition sur le primitivisme, par laquelle j'ai été ébloui. Bien sûr, j'avais, comme tous les spécialistes, des critiques, mais l'exposition, de par les œuvres montrées, était formidable. On y voyait des pièces magnifiques et des rapprochements intéressants. Mais selon moi, on pouvait y opposer deux critiques majeures. La première est que l'exposition était vraiment formidable pour la partie historique classique, du début du xxe siècle jusqu'à la Seconde Guerre mondiale, mais lorsqu'on arrivait aux contemporains, c'était une catastrophe. C'était comme si les commissaires n'avaient pas tenu compte de l'art européen. Je ne serais d'ailleurs pas étonné que Rubin n'y ait pas attaché une grande importance en vérité. Lui s'est concentré sur Picasso, sur le cubisme, sur ce qui est sa spécialité. On sait que c'est Kirk Varnedoe qui s'est occupé du contemporain. Rubin a dû déléguer. Il ne s'est pas rendu compte, sans doute, de la manière dont cela allait être considéré. En tout cas, cela allait être jugé par des Européens, et mal, parce que dans cette exposition, on avait bien inclus, par exemple, une figure majeure comme Beuys, mais il était représenté par une œuvre mineure. En outre, bien d'autres artistes européens manquaient à l'appel. Donc, il y avait vraiment là quelque chose qui ne marchait pas et, dans ce sens, Magiciens de la terre complétait Primitivism. La seconde critique est plus interne, ou plus idéologique. Je n'ai jamais pu accepter la déclaration de Rubin selon laquelle il n'avait jamais mis les pieds en Afrique et que ça ne l'intéressait pas. Rubin se rangeait ainsi derrière Picasso. Picasso l'avait dit, il pouvait le dire. On peut être historien de l'art et ne pas être anthropologue mais tout de même, à ce point-là, c'est un peu gros. Évidemment, je me trouvais à l'opposé de toutes ces idées. Il y a également eu le débat lancé par Mac Evilley dans Artforum. Ce qui est assez amusant, c'est que je me suis trouvé pris dans ce débat lorsque Tom Mc Evilley a attaqué très justement Rubin sur le fait qu'il prétendait avoir été le premier à juxtaposer art moderne et art primitif. Or, nous
Fig. 2 Samuel Kane Kwei, Oignon vert, 1993 , peinture émail sur bois $(88 \times 270 \times 33 \mathrm{~cm})$. Courtesy CAAC The Pigozzi Collection, Geneva @ Kane Kwei.

\footnotetext{
-

6. Primitivism in the $x x^{\text {th }}$ century, 27 septembre $1984-15$ janvier 1985, Museum of Modern Art, New York.
} 
avions élaboré avec Pontus Hulten, à l'ouverture du Centre Georges-Pompidou en 1977, dans les collections permanentes, deux vitrines très importantes avec des objets que le musée de l'Homme nous avait prêtés. L'une, sur l'Afrique, se trouvait en face des cubistes et l'autre, sur l'Océanie, en face des surréalistes. Je recevais des coups de fils à 3 heures du matin de Rubin m'interrogeant sur le nombre d'œuvres qu'il y avait dans cette vitrine, demandant s'il y avait des photos. Évidemment, dans la folie de l'ouverture, on avait oublié de prendre en photo tout cela. Mais je savais que le nombre d'œuvres s'élevait à quelques dizaines. Chacun voulait savoir s'il pouvait argumenter pour discréditer l'autre.

J'avais même écrit un petit texte à l'époque, que l'on distribuait sur des feuillets remis aux visiteurs. J'y expliquais que ce n'était pas uniquement pour des rapprochements formels entre les arts de ces cultures et le modernisme et l'influence qu'ils avaient pu avoir etc., que ces œuvres se faisaient face, mais tout autant pour prouver l'existence, dans le monde, de création artistique en dehors du modernisme. On était là tout de même assez loin de Rubin. Lorsque je suis allé voir Rubin au moment de Primitivism in the $\mathrm{xx}^{\text {th }}$ century, je ne le connaissais pas bien et je voulais lui parler de mon projet. Je lui en ai dit quelques mots, mais j'ai à peine eu le temps de terminer trois ou quatre phrases qu'il m'a conseillé d'aller voir son collaborateur du MoMA, le spécialiste en charge des relations avec l'étranger et en particulier avec l'Amérique du Sud. En vérité, Rubin n'avait pas voulu entendre, ça ne l'intéressait pas. Cela ne faisait pas du tout partie de sa sphère intellectuelle, ni de ses questionnements. Cela aurait pu être intéressant s'il avait accepté de dialoguer, mais ce ne fut pas le cas.

CARLO SEVERI : Avant de parler de conflits culturels et de rapport avec l'anthropologie, je voulais revenir sur ce qui me semble un point essentiel de votre travail. Vous écrivez : "Bâtir une exposition, c'est associer mentalement des œuvres." Je tente de prolonger votre pensée : il s'agit de bâtir un espace de pensée où, par hypothèse, des œuvres établissent un échange. Ce travail sur l'espace intermédiaire apparaît, me semble-t-il, au château d'Oiron et dans Artempo. Pour moi, Artempo, c'est une exposition dans laquelle on thématise la relation entre les œuvres encore plus que les œuvres en elles-mêmes. Ce moment-là, pour moi, a constitué presque un point de bascule, en ce sens que c'est la première exposition qui radicalise le type d'espace intermédiaire qu'on peut bâtir grâce au dialogue entre les œuvres. Le travail de la pensée visuelle est là. Voulez-vous nous parler un peu de cette exposition, de cette manière d'associer les œuvres?

JeAN-Hubert Martin : Il y a plusieurs points. D'abord, avant Artempo, vous citiez le château d'Oiron. C'est tout à fait juste. Là, je maintiendrai une continuité. Mais il y a un maillon essentiel qui manque entre les deux. Il a été vu par beaucoup moins de gens, parce que c'était à Düsseldorf. C'est ce que nous avons appelé le Künstler Museum au Museum Kunst Palast dont j'ai pris la direction en 1999-2000 et qui a ouvert en 2001. C'était une réinstallation, un ré-accrochage de la collection que j'ai réalisé avec deux artistes selon un ordre qui n'était défini ni par les techniques des œuvres ou par leurs supports, ni par la chronologie. J'ai pensé que des artistes pouvaient tout à fait adhérer à mes idées, et cela a été le cas. Donc, j'ai un peu délégué. On a fait ça à trois, avec Thomas Huber et

7. «Musée des charmes», Künstlermuseum. Bogomir Ecker-Thomas Huber. Düsseldorf, Museum Kunst Palast, 2002 : 7-40.
Bogomir Ecker, deux artistes de Düsseldorf, l'un peintre, l'autre sculpteur. On a réalisé un accrochage complètement transversal7. Mélanger les périodes me tenait à cœur depuis très longtemps et on ne peut mener à bien un tel projet que 


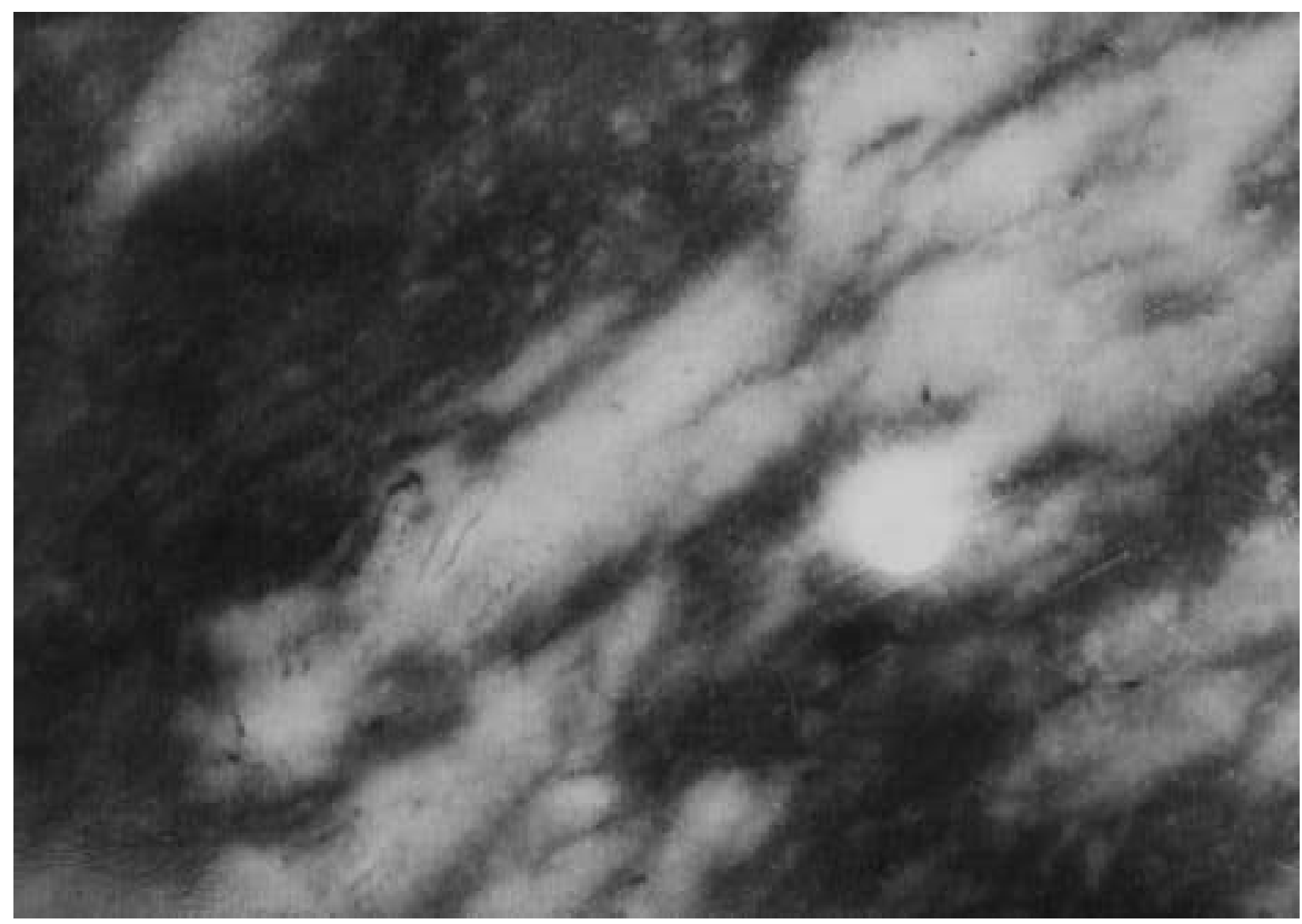

Fig. 3 Vik Muniz, Equivalents (Museum of Modern Art), 1995, tirage photographique sur gélatine d'argent monté sur une planche du musée $(35,5 \times 27,9 \mathrm{~cm})$. Collection de l'artiste @ ADAGP, Paris 2011, Photo courtesy Vik Muniz Studio et Galerie Xippas. 
lorsqu'on dispose d'un musée encyclopédique, qui va de l'Antiquité à nos jours. C'est une idée qui remonte, pour moi, au début des années 1980, mais là, je disposais de matière avec la collection de ce musée des Beaux-Arts. On a donc osé des rapprochements assez étonnants, inédits, surprenants. Il y avait des thèmes relativement conventionnels : on a regroupé des natures mortes ou des portraits, ce qui n'avait rien d'extravagant. Mais déjà, pour les historiens d'art, ça posait un problème: plusieurs de mes conservateurs à l'époque se sont déclarés en porte-à-faux par rapport à ce projet. L'une des conservateurs qui s'occupait de peintures anciennes me disait par exemple que ça n'avait aucun sens de mettre ensemble des paysages européens, allant $d u \mathrm{xvI}^{\mathrm{e}}$ au xix siècle puisque c'était des conceptions complètement différentes. Je répondais que justement, il serait peut-être intéressant de comparer ces interprétations hétérogènes. Il y a quand même une sorte de continuité dans l'histoire du paysage, avec des ruptures,

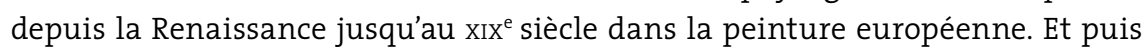
on a établi des rapprochements beaucoup plus intéressants. On a pris des idées, des questions d'aujourd'hui en se demandant comment y répondre avec des objets, des œuvres appartenant à l'histoire, et en les prenant non pas parce qu'ils sont anciens et qu'ils ont une valeur historique importante pour nous, mais pour répondre à ces questions-là. Nous sommes arrivés à faire deux ou trois regroupements qui étaient vraiment excitants, je crois. Ce qui m'a surtout plu dans ce travail avec des artistes, c'était la manière dont ils regardaient les œuvres, parce qu'ils se projetaient immédiatement dans la peau de l'auteur, quelle que soit la période à laquelle appartenait l'objet. Et là, une espèce d'imagination fleurit, ce qui est formidable, et dont souvent les historiens d'art font l'économie, à tort. Parce qu'après tout, on est là pour interpréter et les interprétations peuvent prendre de nombreuses directions. Ces espaces intermédiaires dont vous parlez doivent toujours tenir compte du pareil et du distinct. Ils peuvent être linéaires et relier deux objets. Leur distinction doit être significative. L'exercice devient plus stimulant lorsqu'il s'agit de triangulation et que chaque objet apporte sa plusvalue de sens.

CARLo SEVERI : Je crois que résident là des éléments importants dont vous ne parlez qu'à mi-mots et que l'on peut lire dans certains de vos textes. Dans un autre entretien, vous déclarez : «Pour beaucoup d'historiens de l'art, la communauté qui légitime leur travail, ce sont les autres historiens de l'art, tandis que moi, ma communauté de légitimité, ce sont les artistes. " En effet, cette idée de s'attacher à l'ordre des œuvres et de bousculer le concept de chronologie est au cœur du travail d'un certain nombre d'artistes. Le premier qui me vient à l'esprit, c'est Jiri Kolaj, mais on peut penser aussi à d'autres, comme Parmiggiani ou Dimitrijevic qui ont toujours affirmé que, pour eux, il n'y a pas d'histoire de l'art. Il me semble que vous réalisez une espèce d'utopie poétique ici qui représente à la fois un enjeu intellectuel pour l'histoire de l'art et sa chronologie et une interrogation sur les relations qu'elle peut entretenir avec l'anthropologie. Accepte-t-on tels quels, sans discuter, les critères de l'histoire de l'art, ou ne peut-on pas imaginer une géographie plutôt qu'une histoire, ou une chronologie des œuvres?

Mais revenons à ces situations où la confrontation entre des œuvres qui viennent d'horizons différents - on est toujours dans l'univers de la pensée visuelle, dans son lieu d'exercice - marque un conflit. Nous sommes alors, comme dans Magiciens de la terre, ou comme à la Biennale de Lyon, en train de mettre sur pied un espace où le conflit se réalise et se donnne à voir. On vous a sans doute 
demandé à de nombreuses reprises de reconstruire la continuité entre Magiciens de la terre et les expositions qui en ont pris la suite. Sur ce thème, je vous poserai une question un peu différente. Dans Magiciens de la terre, à Lyon ou ailleurs dans Africa Remix 8 par exemple, vous mettez en coprésence l'art contemporain non occidental avec les recherches européennes ou américaines, dans des lieux où on ne l'attendait pas. Mais vous avez aussi organisé deux expositions où le jeu est encore plus compliqué. Il me semble que dans Altären' (l'exposition consacrée à l'espace de l'autel que vous avez montée à Düsseldorf) et dans Art that Heals ${ }^{10}$ on évoque aussi des espaces rituels. Dans ces deux cas, la pensée visuelle est donc à l'œuvre dans un espace tout à fait différent. Ce décalage vous parait-il intéressant? Ne pourrait-on pas rapprocher cela de ce que vous dites dans une polémique autour d'Africa Remix : "Ah, si je pouvais transférer la pensée des Autels et la considérer comme une œuvre contemporaine, ce serait là un succès?»

JeAn-Hubert Martin : Oui. Je pose même la question : est-ce que c'est de l'art ou pas? Pour moi, le sous-entendu est clair. Cela en est et je me demande pourquoi cela n'en est pas pour le système intellectuel dans lequel nous vivons. Pourquoi n'y a-t-il aucun champ où cette activité d'une richesse formelle incroyable, d'une diversité invraisemblable soit conservée? Quand on a fait Altären, Philippe Peltier a effectué une petite recherche et, aussi incroyable que cela puisse paraître, il n'existait aucun livre sur les autels.

CARLO SEVERI : Une exposition de ce type est donc pour vous une sorte d'expérimentation. Il s'agit d'exposer un autel dans un lieu de l'art pour montrer quelles sont les différences et quelles sont les ressemblances dans la mobilisation du regard que ces objets suscitent. Or, avec Julien Bonhomme, nous en venions à réfléchir, non pas sur la question du statut de l'artiste en Occident et ailleurs, puisque c'est une question sur laquelle vous vous êtes déjà expliqué plusieurs fois et dont vous avez fini par dire qu'il s'agissait d'une question empirique - donc il n'y a rien à figer, c'est bien de la pensée ethnocentrique que de poser cette question-là - mais sur le fait que vos propres travaux, vos propres initiatives parfois transforment quelqu'un en artiste.

Julien BonноммE : Je pensais notamment à Fréderic Bruly Bouabré qui représente un bon exemple de métamorphose de l'artiste. Bouabré a vraiment un parcours intéressant qui interroge ce qui est de l'ordre de l'art et ce qui est de l'ordre du rituel. Il commence en effet sa carrière comme prophète. Il fonde un culte, L'Ordre des persécutés, mais son culte rate, puisque son mouvement prophétique ne prend pas vraiment au-delà de son village d'origine. Il échoue dans l'ordre prophétique et c'est alors qu'il devient un inventeur d'écriture, en créant son extraordinaire syllabaire bété. C'est à partir du travail créateur d'un Bouabré à la fois écrivain et inventeur d'écriture, qu'il va pouvoir naître comme artiste, au fur et à mesure que s'autonomisent les dessins qui servent de supports à son syllabaire. Il va en effet devenir, à la faveur des événements, au nombre desquels Les Magiciens de la terre jouent un rôle important, un artiste à part entière. Plusieurs expositions lui sont alors consacrées. Le cas de Bouabré est intéressant parce qu'on voit qu'il suit de manière obstinée tout au long de sa vie, un même fil directeur qu'il recherche depuis L'Ordre des persécutés, au début des années 1950 jusqu'à son assomption comme artiste dans les années 1990.

\footnotetext{
$0 \%$

8. Africa Remix. L'art contemporain d'un continent, 25 mai-8 août 2005, Centre Georges-Pompidou, Paris.

9. Altären, 9 septembre 2001 6 janvier 2002, Museum Kunst Palast, Düsseldorf.

10. Art that Heals, 6 mars6 avril 2002, Apexart, New York.
} 
JEAN-HubERT MARTIN : Bruly Bouabré l'explique lui-même très bien, avec une formule tout à fait limpide. Il dit : «Je pensais devenir Victor Hugo, je suis devenu Delacroix. "Vous dites, je crois, que sans Magiciens de la terre, cela aurait encore pris beaucoup de temps. Je ne dis pas que quelqu'un ne l'aurait pas découvert en tant que dessinateur, mais nous étions vraiment pionniers. Vous devez connaître l'anecdote d'André Magnin qui était en Côte-d'Ivoire, sur le point de reprendre l'avion, lorsqu'on lui annonce qu'il y a encore quelqu'un à voir. Et il tombe sur Bouabré. Il revient avec ses dessins et on s'extasie tous en disant que c'est formidable. Des anthropologues canadiens avaient déjà publié plusieurs textes de Bruly Bouabré, ces textes étaient agrémentés de quelques petits dessins, en noir et blanc, placés en bas de pages. Mais je crois que, tout de même, l'exposition Magiciens de la terre a beaucoup compté pour sa reconnaissance. Effectivement, de prophète, il est devenu artiste et il a rapidement assumé cette fonction avec beaucoup d'habileté.

Lorsqu'on choisit l'auteur d'objets de culture matérielle dans une autre culture et un autre continent, et dans une société et une culture qui ne connaît pas le rôle social de l'artiste tel qu'il existe chez nous, et qu'on l'appelle artiste, que l'on en fait un artiste, est-ce que l'on agit de manière extravagante? C'est une problématique qui m'intéresse beaucoup, et dont j'ai longuement débattu. Je ne crois pas que ce soit extravagant parce que, là, on touche à des problèmes qui sont de l'ordre du nominalisme. L'auteur s'appelle-t-il un artiste ou pas? Je me suis fait admonester, dans un colloque, à Accra, par pleins de gens, Occidentaux compris, qui, évidemment, suivaient les Noirs dans le mouvement. Je me faisais sermonner - ça ne portait pas sur Bruly Bouabré, mais c'est presque la même chose - parce que j'avais dit que Kane Kwei, l'inventeur des cercueils historiés (phantasy coffins), était un artiste. La question de la paternité et de l'invention a été débattue par plusieurs auteurs. Kane Kwei se l'est attribué, lorsque je l'ai rencontré à Teshie, un faubourg d'Accra en 1988. Quoiqu'il en soit il a développé un genre inventé par lui et d'autres créateurs et l'a fait connaître à l'étranger. Je ne vois pas en quoi il y aurait un préjudice néocolonial à lui conférer une distinction valorisante pour notre échelle sociale, sinon de s'en tenir à un relativisme radical qui n'est plus de mise à l'âge de la mondialisation. J'ai eu une longue conversation avec Simon Njami à ce propos. Ça a débuté au cours de ce colloque et nous nous sommes toujours promis de la publier. Cela a pris des années et nous avons effectivement eu un échange par mails très approfondi sur cette question. On a décidé, puisque Simon était commissaire de l'exposition Africa Remix, de le publier dans le catalogue de l'exposition. Et donc, dans la version allemande, il y a cet entretien entre lui et moi, consacré à cette question. Dans la version française, Simon Njami l'a fait supprimer. Pour moi, c'était le signe d'une victoire intellectuelle. Vraiment, fait-on quelque chose d'extravagant lorsqu'on prend un auteur dans une culture quelconque et qu'on l'amène ici en tant qu'artiste alors que son statut social n'est pas forcément celui-là dans sa culture?

CARLo SEVERI : Il y a d'une part une idée dada de l'artiste, qui serait là pour faire bouger la limite du concept d'art lui-même. Et d'autre part, une position de l'artiste, beaucoup plus traditionnelle, à laquelle on se réfère quand on parle par exemple de la Renaissance. Quelle est la définition d'artiste à laquelle vous vous référez maintenant?

JEAN-Hubert MARTin : L'artiste est celui qui transpose dans la matière les croyances, les récits et les idées d'une communauté, quitte à les faire évoluer. Je ne voudrais pas non plus que l'on croit qu'il n'y ait que l'option dadaïste qui retienne 


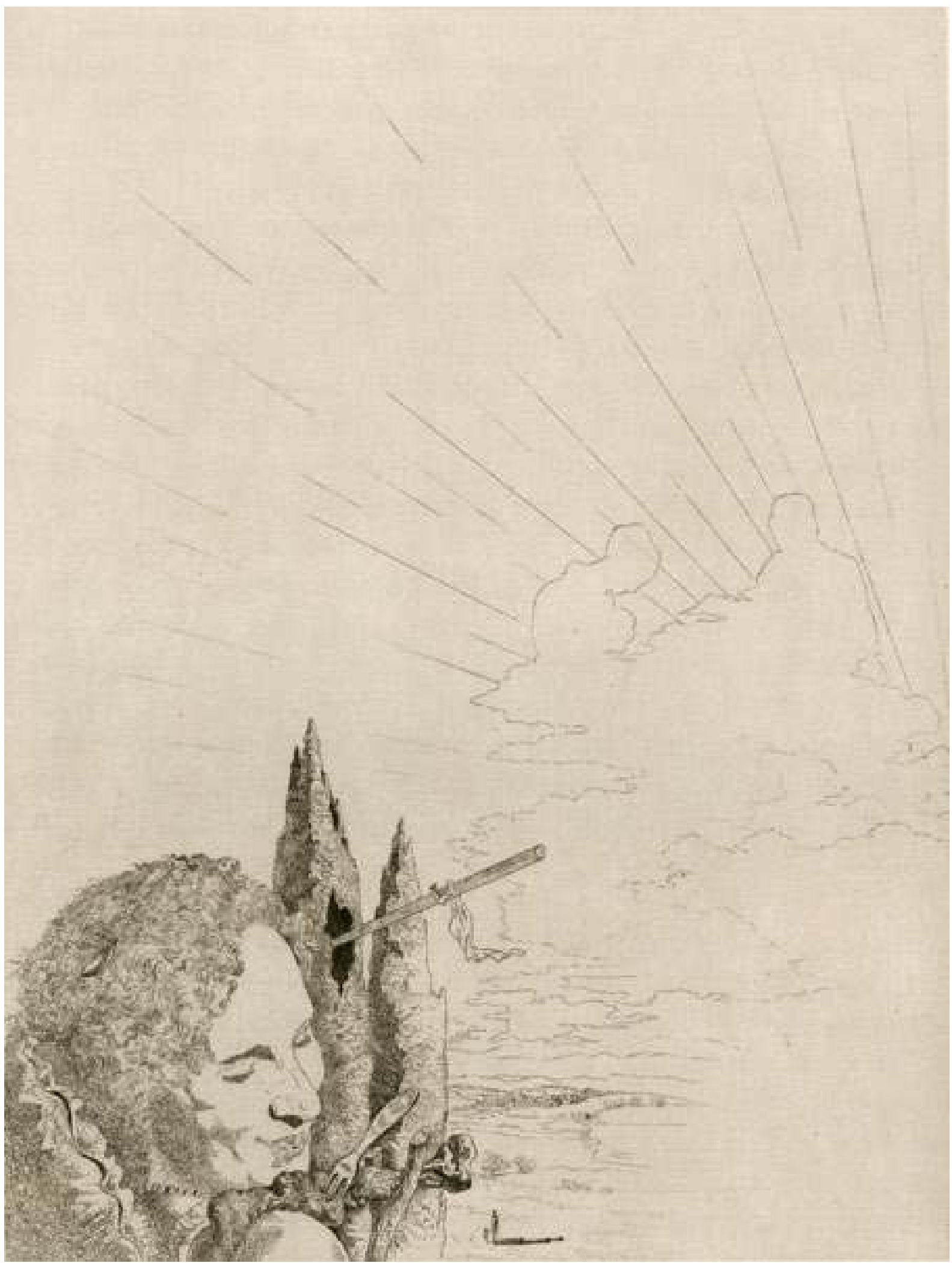

Fig. 4 Salvador Dalí (Figueras), Isidore Ducasse, Albert Skira, L'Angelus anthropomorphique et glaneuse de Millet accompagnés de 1814 de Messionier Planche 22,1934, matière et héliogravures à grains reprises aux outils (indiquées comme eaux-fortes au colophon) (22,4 X $17 \mathrm{~cm})$. Musée d'Art et d'Histoire, Genève @ musée d'Art et d'Histoire, ville de Genève, inv. n E 68-179/22 Photo André Longchamp. 


\section{PIÈGES À VOIR, PIÈGES À PENSER}

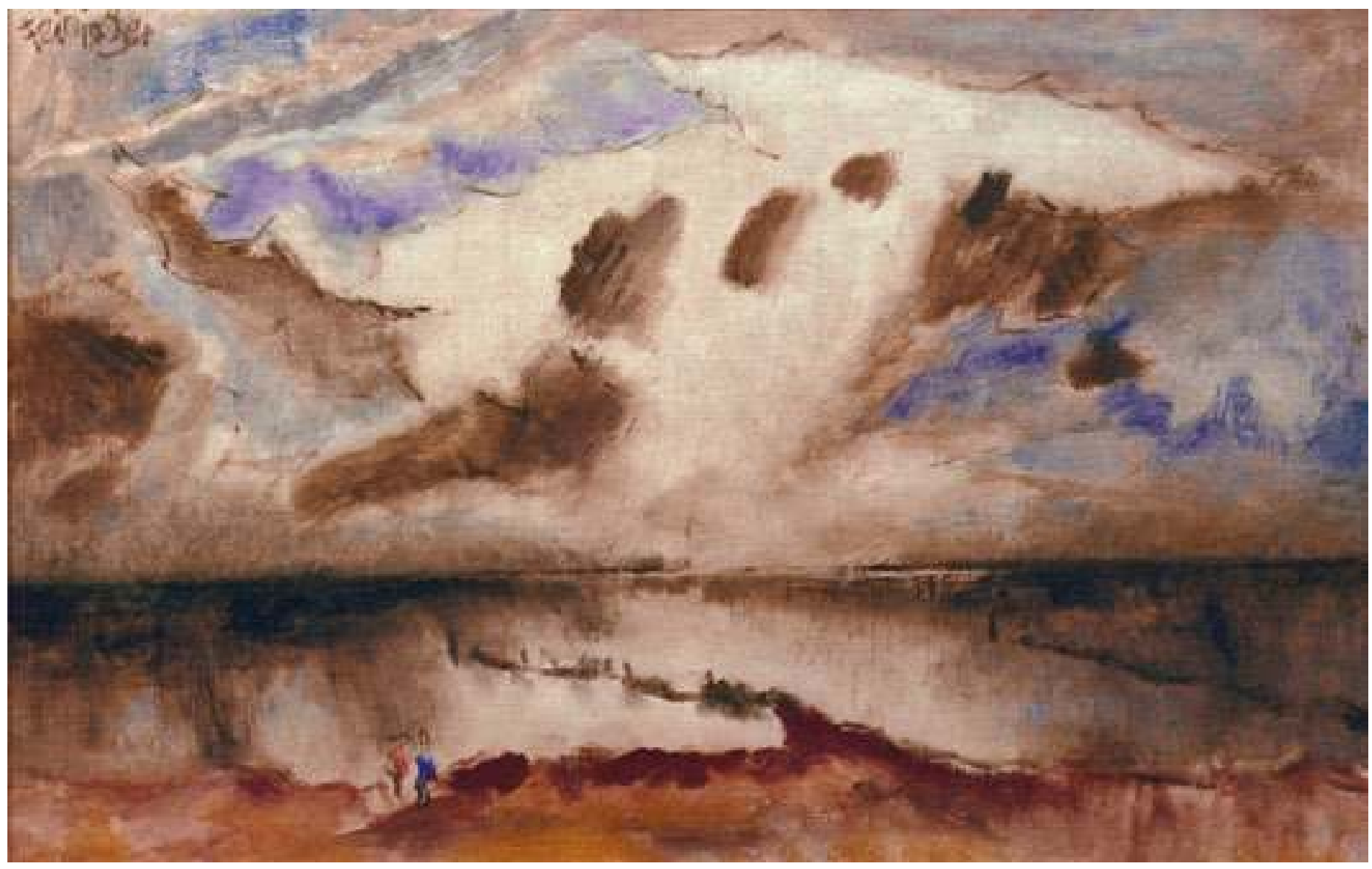

Fig. 5 Lyonel Feininger, The Big Cloud (Große Wolke), 1941, huile sur toile ( 45,8 x $71 \mathrm{~cm}$ ). Collection Marlborough International Fine Art (c) ADAGP, Paris 2011/Photo akg -images. 
mon attention. Il est vrai que je ressens une nette préférence pour l'artiste en tant qu'inventeur d'idées et de formes, qui fait évoluer des formes ritualisées parce qu'à un moment donné il pense que les formes de transcriptions des croyances ne correspondent plus à leur évolution. J'ai une conception assez simple de la modernité. Il y a deux familles, deux familles intellectuelles qui sont tellement imbriquées dans une dialectique que, en vérité, on retrouve les deux idéologies au sein de mêmes individus qui commencent d'un côté et qui terminent de l'autre. D'une part, une pratique de l'art conventionnelle - la peinture, la sculpture - et d'une autre part, les dadaïstes qui font feu de tout bois. Alors, bien entendu, à un moment, tout cela s'affronte en deux clans très évidents. Mais cela se mêle énormément également après. Si l'on prend l'ensemble des déclarations de grands artistes attachés à la peinture, on trouvera certainement des éléments qui sont du côté de cette remise en cause des choses, d'inspiration dadaïste. Cette dialectique est même devenue schématique dans une certaine portion de l'art contemporain où il est quasiment convenu que l'artiste doit commencer par tout péter, faire de la merde, exposer des déchets, puis, dix ans plus tard, faire des œuvres beaucoup plus acceptables, qui passent sur le marché. Et on trouve également le cas inverse d'artistes avec une certaine notoriété qui, vers la fin de leur vie, se remettent en question. Tout d'un coup, on voit arriver un bout de pensée dadaïste où justement ils remettent en cause leur adhésion à des rites et à des conventions qui les rattachent à la tradition. Voilà, il s'agit juste d'expliquer la manière dont je vois les choses, pour ne pas rester uniquement du côté dadaïste. Dadaïste, oui, mais imbriqué dans une dialectique qui est absolument inhérente à la modernité.

CARLo SEVERI : Venons-en à Une image peut en cacher une autre. Restons sur la question du travail de la pensée visuelle. Ici, il consiste à réaliser un espace où les œuvres s'influencent réciproquement, comme ailleurs. Sauf que, là, le jeu est double, puisque les œuvres elles-mêmes sont composées de confrontations d'images différentes. Vous ordonnez ces rapports entre les choses en fonction de certains thèmes. Par exemple, vous avez choisi microcosme/macrocosme, vous avez repéré des rimes visuelles, ou avec Dario Gamboni, les aventures de l'image potentielle, etc. Et puis il y a la rencontre avec des arts non occidentaux, l'art moghol, par exemple. J'étais curieux de comprendre comment vous avez travaillé à cela, c'est-à-dire comment était née l'idée d'imaginer un espace pour ces disciplines.

JEAN-Hubert MARTin : Oui, l'art moghol est une surprise, cela a été une découverte, qui ne se trouvait pas du tout à cet endroit à l'origine, dans la première version que j'avais imaginé de l'exposition. C'est né du surréalisme, de ce surréalisme qui a fait émerger de l'histoire des artistes que l'on considérait jusque-là comme mineurs, comme Arcimboldo, par exemple, et les a fait connaître, au fil des décennies. Grâce aux surréalistes, ce peintre est devenu aujourd'hui l'une des grandes figures de la Renaissance.

CARLo SEVERI : Il s'agit là d'un surréalisme qui dissémine.

JEAN-Hubert MARTIN : Oui, qui dissémine et qui revisite. Comme le dit Malraux : "L'histoire de l'art se lit à l'envers. Ce sont les formes modernes qui nous aident à comprendre les arts du passé. » Notre regard d'aujourd'hui nous permet de faire émerger des œuvres et des artistes. Je suis curieux, je n'aime pas m'ennuyer avec des stéréotypes. J'adore aller rechercher, refaire surgir des oublis. Alors, comment 
est-ce né ? Plutôt du côté du surréalisme et de ce regard surréaliste sur le passé. À partir de ce moment-là, tout d'un coup, on se met à creuser et on voit que le champ devient de plus en plus vaste. Cette exposition s'est tenue en deux temps, puisqu'il y a eu une première version qui s'appelait Dali ou les magiciens de l'ambiguïté, à Düsseldorf. Comme j'étais directeur du musée, position à la fois intéressante et frustrante, il m'a fallu déléguer à un commissaire. Je n'arrêtais pas de le nourrir avec des idées, on discutait, etc. Et puis, j'ai vu qu'on était en train de tirer des fils de tous les côtés, que l'on pouvait continuer à tirer mais qu'il n'avait plus le temps. De mon côté, je manquais également de temps, en tant que directeur, pour effectuer des recherches. On a donc organisé une première exposition, qui a été un vrai succès, et je me suis dit qu'il fallait la réitérer. Après Dali ou les magiciens de l'ambiguité, j'ai eu deux ans pour travailler réellement à Paris. Le livre de Dario Gamboni est sorti au moment où mon exposition s'ouvrait. L'exposition terminée, j'ai lu son livre et je lui ai téléphoné, parce qu'il me semblait qu'il y avait une exposition formidable à monter avec lui, justement en tirant les fils un peu plus loin. C'est d'ailleurs sans fin, j'en trouve toujours de nouveaux.

CARLo SeVtri : C'est une exposition qui suppose un exercice du regard. Les gens acquièrent très rapidement une certaine aptitude à regarder, qui leur fait déchiffrer les images.

JULIEN BonHOMME : Oui, si bien que, effectivement, on peut toujours trouver ces images partout. Par exemple, les tâches de Rorschach, les images dans les nuages, ou bien encore, les rochers anthropomorphes.

JeAn-Hubert Martin : Oui, mais attention, il s'agit là de l'image potentielle. C'est le côté Dario Gamboni. De mon côté, j'ai écarté beaucoup de choses que lui trouvait passionnantes, parce que j'ai toujours voulu m'en tenir à l'ambiguïté, c'est-à-dire à l'image double. Il faut bien distinguer image double et image potentielle. Au sein de notre travail, nous nous interrogions longuement pour freiner certains enthousiasmes et nous concentrer sur la double image.

Julien Bonнomme : Pourriez-vous alors nous éclairer sur les différents sens de cette notion d'image double, de double image, d'image cachée, d'image potentielle? Qu'est-ce qui distingue l'image double de l'image potentielle? Vous distinguez également -il me semble que c'était dans le catalogue - l'image double de la chimère au sens d'image composite.

JeAN-Hubert Martin : Je ne suis plus complètement dans le bain de tout cela aujourd'hui. Mais quant à l'image potentielle, vous voyez bien d'où part Dario Gamboni. Il part d'exemples d'artistes qu'il a beaucoup travaillés, comme Redon, et il va chercher, par exemple, le fait que dans le premier état de telle gravure, vous trouvez une image que l'artiste a retravaillée et que vous ne voyez presque plus dans l'état définitif. C'est un processus de création de l'image, c'est passionnant. Et dans l'œuvre aboutie, il faut quelquefois tout le travail de décryptage de l'historien d'art pour retrouver, comme un palimpseste, ce qu'a été l'origine. L'image potentielle est une suggestion proposée au regardeur. L'exposition, quant à elle, privilégiait la double image, c'est-à-dire l'ambiguïté visuelle, le stade où l'artiste crée consciemment une forme qui peut contenir plusieurs sens, plusieurs interprétations. 
Julien Bonhomme : Toutes ces images doubles supposent un acte de regard. L'acte de regard est pleinement constitutif du fait que ce soient des images doubles. Or, pour certaines, elles sont doubles lorsqu'elles sont vues par un regard distrait. Reprenons la notion de double image, dont vous dites qu'elle vient de Dalí. Je le cite : « La double image apparaît si l'on regarde pendant quelque temps avec un léger recul et une certaine fixité distraite. " En revanche, certaines images doubles supposent un tout autre régime de regard, celui du regard savant, capable de déchiffrer, de lire l'image et d'y lire des intentions cachées, selon une symbolique qui peut se révéler extrêmement complexe. Il y a là une tension intéressante. Si toute image double suppose un acte de regard, celui-ci peut se situer aussi bien du côté d'un regard flottant et distrait, ou au contraire du regard savant qui, lui, est capable de déchiffrer ce qui se trouve derrière l'image.

JEAN-HUBERT MARTIN : Je ne vois pas de réelle opposition dans ce que vous dites là. Le fait du regard distrait, c'est encore le niveau phénoménologique, le niveau du regard. Cela ne veut pas dire qu'une fois que le regard distrait a révélé la double image, il n'y ait pas toutes les intentions symboliques et la complexité intellectuelle que vous citez dans le second cas. Ça ne me paraît pas exclusif parce que, justement, cette complexité, elle existait chez Dalí et c'est lui qui parlait du regard distrait. Le texte de Catherine Millet à ce propos, "Dalí et moi», est passionnant.

Julien Bonhomme : À propos de ce que vous disiez au sujet des spectateurs, du fait que certains sont plus aptes à voir ces images doubles, à les déceler dans l'image, on peut se demander quel type de regard cela suppose, dans quel type de disposition il faut se placer, pour voir plus ou moins bien ou plus ou moins rapidement ces images-là. C'est l'articulation entre perception et projection qui permet de faire apparaître cette double image.

JeAN-Hubert MARTIN : Il y a des travaux sur la question de la perception au niveau cérébral, neurophysiologique. On a fait intervenir Alain Berthoz, du Collège de France, qui a donné une conférence très intéressante à ce propos. Pour le dire de façon simplifiée, il n'y a pas simplement perception, analyse et identification, mais la perception passe par le filtre d'une reconnaissance de formes accumulées au fil de l'éducation et de l'expérience livrant une identification automatique avant l'analyse.

On a dit assez souvent que les enfants étaient beaucoup plus aptes à les voir. J'ai également rencontré des gens qui se rendent à peine dans une exposition, qui n'ont pas du tout de connaissances en histoire de l'art, mais qui voyaient tout de suite, et d'autres, au contraire, qui sont dans l'art depuis longtemps mais qui ne voyaient pas.

CARlo SEVtri : Ce qui est intéressant dans cette exposition, c'est que l'ensemble des œuvres et des choix ne se réduit pas à une doctrine. Cela me parait caractéristique de ce que vous faites. Vous posez dans l'exposition un échiquier d'œuvres qui impliquent des questions importantes - il est évident que pour n'importe quelle image la relation entre perception et projection est importante. L'intérêt de l'image double, c'est qu'elle montre ce processus, d'une certaine manière. Donc, à partir de là, effectivement, ce flottement entre le savant et le distrait s'avère crucial. 
Fig. 6 La harpiste Fée du Shah Bahram-Gour sur un dromadaire composite, $x \mathrm{xII}{ }^{\mathrm{e}}$ siècle, gouache et or sur papier $(23,5 \times 14,5 \mathrm{~cm}$ image et $40 \times 28 \mathrm{~cm}$ feuille). Musée d'Art islamique, Berlin (c) BPK, Berlin, Dist. Rmn/ Georg Niedermeiser.
JEAN-Hubert MARTIN : On en revient à la pensée visuelle, c'est-à-dire que tout objet est digne d'être regardé et peut apporter des éclaircissements incroyables, des points de vue, des rapprochements. Il se tisse des constellations, et ça ne concerne pas exclusivement ce qui entre dans la catégorie de l'art.

CARLo SeVEri : Ce que vous dites est intéressant si l'on revient à ce que vous proposez d'appeler avec une jolie formule, «l'art primitif (sic)». Vous avez surtout travaillé dans le domaine du visuel. Or, souvent dans «l'art primitif (sic) », le visuel est indissociable d'autres dimensions qui, précisément, permettent ces rebondissements. Il y a quelques années, j'avais analysé une harpe zandé - c'était après avoir vu Afrique. Formes sonores ${ }^{11}$, une très grande exposition au musée national des Arts africains et océaniens - et je m'étais rendu compte que ces têtes sculptées sur un support qui produit un son confèrent virtuellement à la musique le statut d'une voix. Le visuel et le sonore étaient dans une situation d'interpénétration inconnue de la tradition occidentale. Ne pourrait-on pas imaginer une nouvelle exposition qui, au lieu de miser seulement sur le fait visuel, pourrait identifier d'autres axes, dans l'action et la performance? Les artistes contemporains ont bien vu cela également. Si ce sont eux qui doivent légitimer notre travail et je suis tout à fait d'accord avec ce postulat, ne pourrait-on pas penser à une exposition Magiciens de la terre II, la performance? On pourrait alors imaginer des séquences d'actions : Lebel, Abramovic, Beuys, etc., confrontées avec des séquences d'actions non occidentales...

JeAN-Hubert MARTIN : Immédiatement, je me pose une question très pragmatique : quand vous dites faire une exposition avec des performances, s'agit-il des performances réelles, et donc cela se passe dans le temps et cela se succède sans doute, ça ne peut être simultané, ou est-ce que ce sont des vidéos? Visuellement, dès que c'est sur vidéo, moi, ça ne m'excite jamais beaucoup. Dans l'exposition sur les autels, j'avais adjoint à la plupart une petite vidéo montrant leur usage rituel où le son jouait un rôle essentiel.

CARLO SEVERI : Je cherchais par cet exemple à moitié imaginaire à mieux définir une question qui me semble essentielle. Je vais donner un exemple : un jeune ethnologue, Arnaud Morvan, dans les recherches qu'il a pu mener en Australie, a eu à la fois la sensibilité et la chance de se trouver, pour sa thèse, au sein d'un groupe d'artistes formidables. Il a été accepté, il a partagé leur vie, a travaillé avec eux. Ce groupe d'artistes a peint au moins six grandes toiles qui sont une mémoire d'un massacre colonial. Or, ces artistes ont également inventé un chant pour accompagner les toiles. Arnaud a eu la possibilité de prendre connaissance des deux. Néanmoins, très souvent, quand les œuvres de ce type partent pour une galerie ou un musée occidental, les chants restent. Ne pourrait-on pas imaginer un jour de réfléchir au fait qu'il y a là une articulation entre la parole rituelle et l'image, qui fait que l'œuvre est composée des deux? Ce n'est pas du tout pour nier le parcours que vous avez accompli dans la dimension du visuel, mais plutôt pour tenter d'en imaginer une démultiplication.

JEAN-Hubert MARTin : Beaucoup d'œuvres sont le résultat de rituels. Ça s'est fait dans l'art contemporain récent. Il y a eu une exposition, que je n'ai pas vue, qui traitait de l'action painting. À partir des années 1960, plus encore qu'auparavant, a éclos une multitude d'œuvres, dans l'art contemporain, totalement inséparables du rituel, même personnel, qui les a produites. 


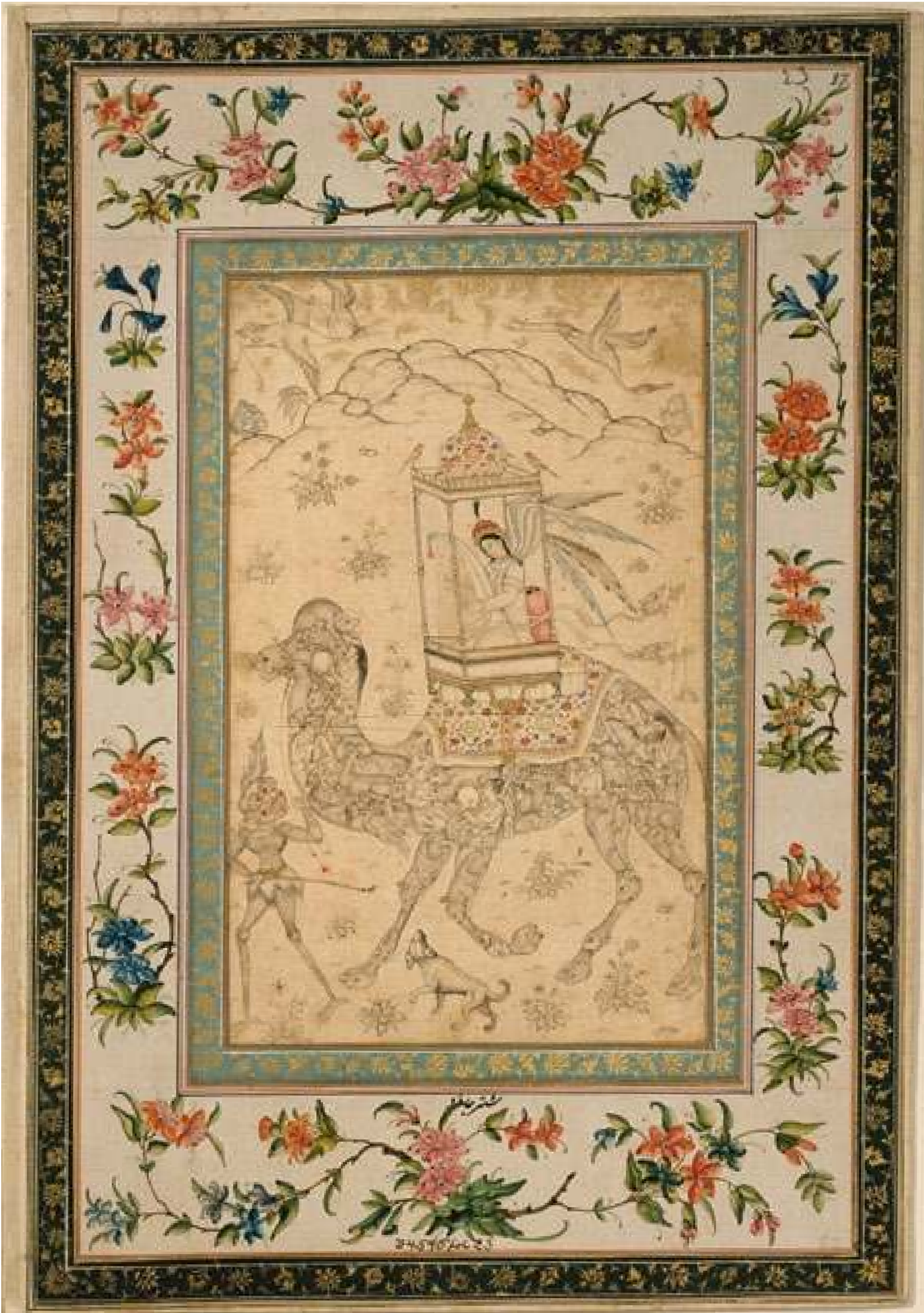


CARLo SEVERI : Je pensais aussi à Artempo où vous avez exposé Opalka. Un artiste qui, face à la toile, fait entendre sa voix, récite des nombres. On a là une image sonore, qui est loin d'être absente des arts non occidentaux. L'image sonore pourrait donc être une autre piste. Il y a là quelque chose qui pourrait fonctionner comme répondant à une certaine écoute de l'«art primitif (sic)", une manière d'enrichir notre perspective, qui nous permettrait de saisir cette dimension-là.

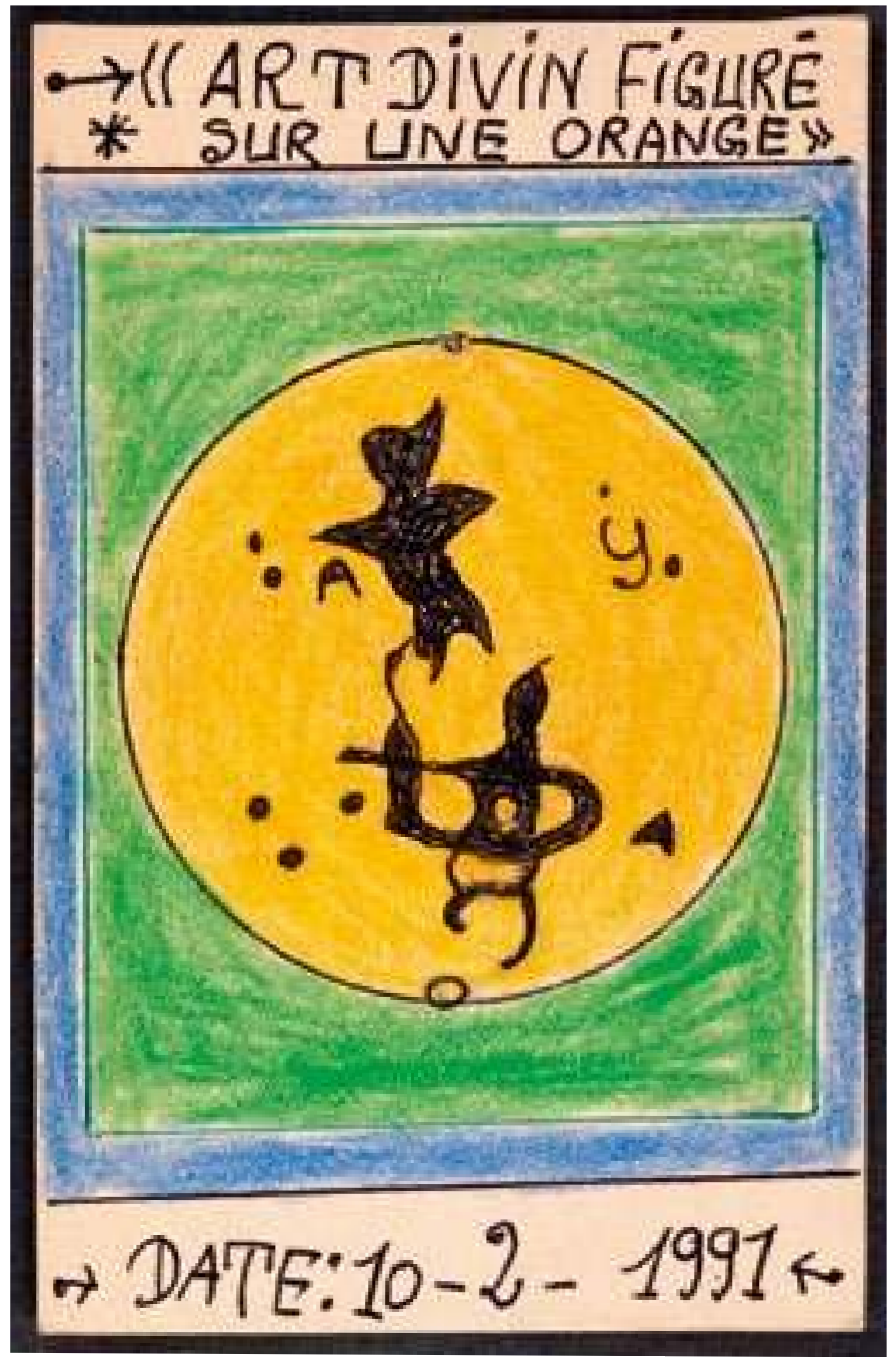

JEAN-Hubert MARTIN : Il y a l'art occidental, mais le Japon joue également un rôle important. Gutaï, c'est exactement ça. L'essentiel de Gutaï, c'est le résultat d'actions, quelquefois violentes. La peinture de Shiraga, ce n'est pas autre chose.

Carlo Severi : C'est vous qui m'avez mis sur cette piste, je ne connaissais pas Shigara. Après, j'ai découvert ses toiles. Effectivement, dans ce cas, la peinture est le précipité d'une action.

Jean-Hubert Martin : Et puis Yves Klein. Sans doute a-t-il tout fait pour l'effacer, le masquer, etc., mais il a été influencé par les Japonais. Il s'est débrouillé pour que les traces disparaissent. Mais c'est quelque chose à retravailler. On trouverait beaucoup d'exemples dans d'autres continents, d'autres cultures, très intéressants. D'ailleurs, vous parliez des Aborigènes, j'ai appris récemment que l'un des peintres aborigènes que j'adore, Rover Thomas, avait réalisé ses premières peintures pour un spectacle avec des chants, où il racontait un massacre. J'ai vu des photos où il porte des peintures qui font partie de la pièce de théâtre créée avec sa communauté. Ça ne s'est autonomisé en tant que pratique picturale que bien après.

Fig. 7 Fréderic Bruly Bouabré, Art divin figuré sur une orange, série de 48 dessins "Signes relevés sur des oranges ", 1991, crayon de couleur et stylo à bille sur carton $(15 \times 10 \mathrm{~cm})$ Courtesy CAAC - The Pigozzi Collection, Geneva

๑) Frédéric Bruly Bouabré.
CARLo SEVERI : Donc les images en actes, les images sonores, c'est peut-être un fil sur lequel réfléchir. Maintenant, j'hésite un peu parce que je sais que vous n'aimez pas réduire les expositions, les images, à une doctrine qui amoindrit forcément les ambiguïtés, et par conséquent la richesse des choses. Je vous confie donc simplement quelques réflexions de lecteur, lecteur d'un texte que vous n'avez pas écrit, mais que vous avez suscité par une interview. Vous avez parlé avec Dimitrijevic, au cours d'une longue interview, de cette œuvre extraordinaire qu'est le Triptyque post-historicus. Je vous y ai senti très curieux de ses idées sur l'histoire de l'art. Dimitrievic dit : «Dans l'histoire de l'art, il n'y a pas d'erreurs, c'est l'histoire de l'art qui est une erreur. " Dans votre entretien avec lui, vous réagissez plusieurs fois à cette question. On sent chez vous un sentiment comme une inquiétude qui m'a intéressé. On se dit que Jean-Hubert Martin a en tête le projet 
d'opposer à l'histoire de l'art et à sa chronologie une géographie complexe de l'art, de l'activité artistique. On pense aux bouteilles de schnapps hollandais, trouvées en Afrique au début du xxe siècle, qui seraient contemporaines du porte-bouteille de Duchamp. Auriez-vous le projet de construire une grande vision synoptique de ce qui se passe dans l'art, dans le monde entier? Sans doute. Mais je ne voudrais pas m'arrêter là : cette question de l'espace-temps de l'activité artistique se pose aussi à propos d'Artempo ${ }^{12}$, puisque le thème même de cette exposition était la représentation du temps. On a là l'idée de reposer en termes d'art un problème qui est bloqué dans l'histoire de l'art, autrement dit d'utiliser l'espace de l'art comme une manière de reposer des questions d'épistémologie, de les retravailler par l'exercice de la pensée visuelle.

JeAn-Hubert MARTin : J'ai dit ça à plusieurs reprises dans d'autres termes. Ce qui me préoccupe en tant que praticien des musées, c'est de trouver une autre taxinomie que celle qui m'ennuie. Je ne comprends pas, à la longue, pourquoi chaque musée que je vais voir est ordonné de la même manière. Ce qui d'ailleurs a provoqué, par contrecoup, cette idée fausse qu'au fond on ne peut parler sérieusement aux visiteurs des musées que lorsqu'on leur a inculqué la chronologie - quand Dimitrijevic dit "l'histoire de l'art», il pense "la chronologie». C'est l'erreur fatale. On commence d'ailleurs à s'en rendre compte, je ne suis pas le seul. J'ai eu cette intuition peut-être un petit peu avant certains; et depuis très longtemps, je suis à la recherche d'une nouvelle taxinomie. C'est pour ça que j'ai creusé du côté des cabinets de curiosités. Au cours de conversations avec Patricia Falguières, je me suis dit que, peut-être, il y avait à la Renaissance une taxinomie des œuvres, intéressante et inattendue. Il y avait deux bibles pour moi, les travaux d'Adalgisa Lugli ${ }^{13}$ et ceux de Schnapper ${ }^{14}$, deux ouvrages pionniers qui donnent des pistes sur les différentes thématiques, sur les différentes options du cabinet de curiosités. Cela dit, par rapport à la pensée d'aujourd'hui, l'appel à la pensée de la Renaissance pour réordonner une collection de musée n'a pas de sens. Mais je continue de chercher quelque chose de cet ordre que je n'ai pas encore trouvé. J'ai un projet d'exposition qui me tient beaucoup à cœur et sur lequel je travaille depuis deux ou trois ans. J'espère y arriver. Ce sera une proposition, ça risque d'être pris comme une proposition très subjective, personnelle. Et ce n'est pas une taxinomie. J'ai une idée pour présenter des œuvres de toutes origines de manière autre.

CARLo SeVeri : Un projet d'univers pour ainsi dire.

JeAN-Hubert Martin : Oui, un petit univers.

JeAn-Hubert MARTin : Ce qui me satisfait beaucoup, c'est qu'après des années de travail, je vois avec beaucoup de satisfaction arriver le retour de certaines de mes expositions. Cela s'oublie très vite les expositions. J'ai l'énorme chance que Magiciens de la terre ait bénéficié de la destinée qu'on lui connait. Depuis, je travaille, je corrige, et une exposition en suit une autre. Ce sont des projets qui se poursuivent et dont j'ai souvent l'impression qu'ils ne sont pas perçus. Donc je vous remercie de votre intérêt parce que ça prouve que ce n'est pas en vain.

CARLO SEVERI : Il y a des expositions qu'on oublie. Il y en a aussi que l'on n'oublie pas. Merci.
- $\bigcirc$

12. Artempo: where time becomes art, 9 juin-? octobre 200?,

The Fortuny Museum, Venise, cf. «About Artempo:

a conversation » (entretien avec Axel Vervoordt ], in Artempo : where time becomes art, catalogue de l'exposition éponyme. Gravenwezel-Gand, Axel VervoordtMer. Paper Kunsthalle, 2007 : 25-32.

13. Adalgisa Lugli, Naturalia et Mirabilia: les cabinets de curiosités en Europe. Traduit de l'italien par Marie-Louise Lentengre. Paris, Adam Biro, 1998.

14. Antoine Schnapper, Le Géant, la Licorne, la Tulipe. Paris, Flammarion, 1988. 